\title{
Semantics and Termination of Simply-Moded Logic Programs with Dynamic Scheduling
}

\author{
Annalisa Bossi ${ }^{1}$, Sandro Etalle ${ }^{2,3}$, Sabina Rossi ${ }^{1}$, and Jan-Georg Smaus ${ }^{3}$ \\ 1 Università di Venezia, \{bossi,srossi\}@dsi.unive.it \\ 2 Universiteit Maastricht, etalle@cs.unimaas.nl \\ 3 CWI, Amsterdam, jan.smaus@cwi.nl
}

\begin{abstract}
In logic programming, dynamic scheduling refers to a situation where the selection of the atom in each resolution (computation) step is determined at runtime, as opposed to a fixed selection rule such as the left-to-right one of Prolog. This has applications e.g. in parallel programming. A mechanism to control dynamic scheduling is provided in existing languages in the form of delay declarations.

Input-consuming derivations were introduced to describe dynamic scheduling while abstracting from the technical details. In this paper, we first formalise the relationship between delay declarations and inputconsuming derivations, showing in many cases a one-to-one correspondence. Then, we define a model-theoretic semantics for input-consuming derivations of simply-moded programs. Finally, for this class of programs, we provide a necessary and sufficient criterion for termination.
\end{abstract}

\section{Introduction}

Background. Logic programming is based on giving a computational interpretation to a fragment of first order logic. Kowalski [14] advocates the separation of the logic and control aspects of a logic program and has coined the famous formula

Algorithm $=$ Logic + Control

The programmer should be responsible for the logic part. The control should be taken care of by the logic programming system.

In reality, logic programming is far from this ideal. Without the programmer being aware of the control and writing programs accordingly, logic programs would usually be hopelessly inefficient or even non-terminating.

One aspect of control in logic programs is the selection rule, stating which atom in a query is selected in each derivation step. The standard selection rule in logic programming languages is the fixed left-to-right rule of Prolog. While this rule provides appropriate control for many applications, there are situations, e.g. in the context of parallel execution or the test-and-generate paradigm, that require a more flexible control mechanism, namely, dynamic scheduling, where the selectable atoms are determined at runtime. Such a mechanism is provided in modern logic programming languages in the form of delay declarations [16]. 
To demonstrate that on the one hand, the left-to-right selection rule is sometimes inappropriate, but that on the other hand, the selection mechanism must be controlled in some way, consider the following programs APPEND and IN_ORDER

$\%$ append (Xs, Ys, Zs $) \leftarrow Z s$ is the result of concatenating the lists $\mathrm{Xs}$ and $\mathrm{Ys}$ append $([\mathrm{H} \mid \mathrm{Xs}], \mathrm{Ys},[\mathrm{H} \mid \mathrm{Zs}]) \leftarrow$ append $(\mathrm{Xs}, \mathrm{Ys}, \mathrm{Zs})$. append ( [], Ys , Ys).

$\% \quad$ in_order (Tree,List) $\leftarrow$ List is an ordered list of the nodes of Tree in_order (tree (Label, Left, Right), Xs) $\leftarrow$ in_order (Left,Ls), in_order(Right,Rs), append (Ls, [Label|Rs],Xs). in_order (void, []).

together with the query (read_tree and write_list are defined elsewhere)

$$
\text { q : read_tree(Tree), in_order(Tree,List), write_list(List). }
$$

If read_tree cannot read the whole tree at once - say, it receives the input from a stream - it would be nice to be able to run the "processes" in_order and write_list on the available input. This can only be done if one uses a dynamic selection rule (Prolog's rule would call in_order only after read_tree has finished, while other fixed rules would immediately diverge). In order to avoid nontermination one should adopt appropriate delay declarations, namely

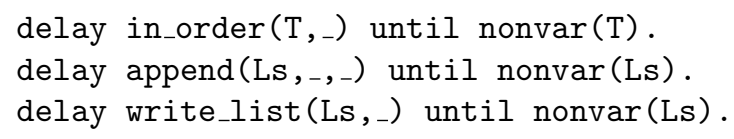

These declarations avoid that in_order, append and write_list are selected "too early", i.e. when their arguments are not "sufficiently instantiated". Note that instead of having interleaving "processes", one can also select several atoms in parallel, as long as the delay declarations are respected. This approach to parallelism has been first proposed in [17] and "has an important advantage over the ones proposed in the literature in that it allows us to parallelise programs written in a large subset of Prolog by merely adding to them delay declarations, so without modifying the original program" [4].

Compared to other mechanisms for user-defined control, e.g., using the cut operator in connection with built-in predicates that test for the instantiation of a variable (var or ground), delay declarations are more compatible with the declarative character of logic programming. Nevertheless, many important declarative properties that have been proven for logic programs do not apply to programs with delay declarations. The problem is mainly related to deadlock.

In the first place, for such programs the well-known equivalence between model-theoretic and operational semantics does not hold. For example, the query append $(\mathrm{X}, \mathrm{Y}, \mathrm{Z})$ does not succeed (it deadlocks) and this is in contrast with the fact that (infinitely many) instances of append $(\mathrm{X}, \mathrm{Y}, \mathrm{Z})$ are contained in the least Herbrand model of APPEND. This shows that a model-theoretic semantics in the classical sense is not achievable, in fact the problem of finding a suitable 
declarative semantics is still open. Moreover, while for the left-to-right selection rule there are results that allow us to characterise when a program is terminating, these results do not apply any longer in presence of dynamic scheduling.

Contributions. This paper contains essentially four contributions tackling the above problems.

In order to provide a characterisation of dynamic scheduling that is reasonably abstract and hence amenable to semantic analysis, we consider inputconsuming derivations [18, a formalism similar to Moded GHC [20]. In an inputconsuming derivation, only atoms whose input arguments are not instantiated through the unification step may be selected. Moreover, we restrict our attention to the class of simply-moded programs, which are programs that are, in a well-defined sense, consistent wrt. the modes. As also shown by the benchmarks in Sec. 6, most practical programs are simply-moded. We analyse the relations between input-consuming derivations and programs with delay declarations. We demonstrate that under some statically verifiable conditions, input-consuming derivations are exactly the ones satisfying the (natural) delay declarations of programs.

We define a denotational semantics which enjoys a model-theoretical reading and has a bottom-up constructive definition. We show that it is compositional, correct and fully abstract wrt. the computed answer substitutions of successful derivations. E.g., it captures the fact that the query append $(X, Y, Z)$ does not succeed.

Since dynamic scheduling also allows for parallelism, it is sometimes important to model the result of partial (i.e., incomplete) derivations. For instance, one might have queries (processes) that never terminate, which by definition may never reach the state of success, i.e. of successful completion of the computation. Therefore, we define a second semantics which enjoys the same properties as the one above. We demonstrate that it is correct, fully abstract and compositional wrt. the computed substitutions of partial derivations. We then have a uniform (in our opinion elegant) framework allowing us to model both successful and partial computations.

Finally, we study the problem of termination of input-consuming programs. We present a result which fully characterises termination of simply-moded inputconsuming programs. This result is based on the semantics mentioned in the previous paragraph.

The rest of this paper is organised as follows. The next section introduces some preliminaries. Section 3 defines input-consuming derivations and delay declarations, and formally compares the two notions. Section 4 provides a result on denotational semantics for input-consuming derivations, first for complete derivations, then for incomplete (input-consuming) derivations. Section 5 provides a sufficient and necessary criterion for termination of programs using inputconsuming derivations. Section [6] surveys some benchmark programs. Section 7 concludes. The proofs have been omitted and can be found in [8]. 


\section{Preliminaries}

The reader is assumed to be familiar with the terminology and the basic results of the semantics of logic programs 11215. Following [2], we use boldface characters to denote sequences of objects: $\mathbf{t}$ denotes a sequence of terms, $\mathbf{B}$ is a query (i.e., a possibly empty sequence of atoms). The empty query is denoted by $\square$. The relation symbol of an atom $A$ is denoted $\operatorname{Rel}(A)$. The set of variables occurring in a syntactic object $o$ is denoted $\operatorname{Var}(o)$. We say that $o$ is linear if every variable occurs in it at most once. Given a substitution $\sigma=\left\{x_{1} / t_{1}, \ldots, x_{n} / t_{n}\right\}$, we say that $\left\{x_{1}, \ldots, x_{n}\right\}$ is its domain (denoted by $\left.\operatorname{Dom}(\sigma)\right)$, and $\operatorname{Var}\left(\left\{t_{1}, \ldots, t_{n}\right\}\right.$ ) is its range (denoted by $\operatorname{Ran}(\sigma)$ ). Note that $\operatorname{Var}(\sigma)=\operatorname{Dom}(\sigma) \cup \operatorname{Ran}(\sigma)$. If $t_{1}, \ldots, t_{n}$ is a permutation of $x_{1}, \ldots, x_{n}$ then we say that $\sigma$ is a renaming. The composition of substitutions is denoted by juxtaposition $(x \theta \sigma=(x \theta) \sigma)$. We say that a term $t$ is an instance of $t^{\prime}$ iff for some $\sigma, t=t^{\prime} \sigma$; further, $t$ is a variant of $t^{\prime}$, written $t \approx t^{\prime}$, iff $t$ and $t^{\prime}$ are instances of each other. A substitution $\theta$ is a unifier of terms $t$ and $t^{\prime}$ iff $t \theta=t^{\prime} \theta$. We denote by $m g u\left(t, t^{\prime}\right)$ any most general unifier (mgu, in short) of $t$ and $t^{\prime}$. A query $Q: \mathbf{A}, B, \mathbf{C}$ and a clause $c: H \leftarrow \mathbf{B}$ (variable disjoint with $Q$ ) yield the resolvent $(\mathbf{A}, \mathbf{B}, \mathbf{C}) \theta$ with $\theta=$ $m g u(B, H)$. We say that $\mathbf{A}, B, \mathbf{C} \stackrel{\theta}{\Longrightarrow}(\mathbf{A}, \mathbf{B}, \mathbf{C}) \theta$ is a derivation step (using $c$ ), and call $B$ the selected atom. A derivation of $P \cup\{Q\}$ is a sequence of derivation steps $Q \stackrel{\theta_{1}}{\Longrightarrow} Q_{1} \stackrel{\theta_{2}}{\Longrightarrow} \cdots$ using (variants of) clauses in the program $P$. A finite derivation $Q \stackrel{\theta_{1}}{\Longrightarrow} \cdots \stackrel{\theta_{n}}{\Longrightarrow} Q_{n}$ is also denoted $Q \stackrel{\vartheta}{\longrightarrow} P Q_{n}$, where $\vartheta=\theta_{1} \ldots \theta_{n}$. The restriction of $\vartheta$ to $Q$ is a computed answer substitution (c.a.s.). If $Q_{n}=\square$, the derivation is successful.

Delay Declarations. Logic programs with delay declarations consist of two parts: a set of clauses and a set of delay declarations, one for each of its predicate symbols. A delay declaration associated with an $n$-ary predicate symbol $p$ has the form

$$
\text { delay } p\left(t_{1}, \ldots, t_{n}\right) \text { until } \operatorname{Cond}\left(t_{1}, \ldots, t_{n}\right)
$$

where $\operatorname{Cond}\left(t_{1}, \ldots, t_{n}\right)$ is a formula in some assertion language [12]. A derivation is delay-respecting if an atom $\mathrm{p}\left(t_{1}, \ldots, t_{n}\right)$ is selected only if $\operatorname{Cond}\left(t_{1}, \ldots, t_{n}\right)$ is satisfied. In particular, we consider delay declarations of the form

$$
\text { delay } \mathrm{p}\left(\mathrm{X}_{1}, \ldots, \mathrm{X}_{n}\right) \text { until nonvar }\left(\mathrm{X}_{i_{1}}\right) \wedge \ldots \wedge \operatorname{nonvar}\left(\mathrm{X}_{i_{k}}\right) \text {. }
$$

where $1 \leq i_{1}<\ldots<i_{k} \leq n 1$ The condition nonvar $\left(t_{i_{1}}\right) \wedge \ldots \wedge \operatorname{nonvar}\left(t_{i_{k}}\right)$ is satisfied if and only if $t_{i_{1}}, \ldots, t_{i_{k}}$ are non-variable terms. Such delay declarations are equivalent to the block declarations of SICStus Prolog [13].

Moded Programs. A mode indicates how a predicate should be used.

${ }^{1}$ For the case that $k=0$, the empty conjunction might be denoted as true, or the delay declaration might simply be omitted. 
Definition 2.1. A mode for a predicate symbol $p$ of arity $n$, is a function $m_{p}$ from $\{1, \ldots, n\}$ to $\{$ In, Out $\}$.

If $m_{p}(i)=$ In (resp. Out), we say that $i$ is an input (resp. output) position of $p$. We denote by $\operatorname{In}(Q)$ (resp. Out $(\mathrm{Q}))$ the sequence of terms filling in the input (resp. output) positions of predicates in $Q$. Moreover, when writing an atom as $p(\mathbf{s}, \mathbf{t})$, we are indicating that $\mathbf{s}$ is the sequence of terms filling in its input positions and $\mathbf{t}$ is the sequence of terms filling in its output positions.

The notion of simply-moded program is due to Apt and Etalle [3].

Definition 2.2. A clause $p\left(\mathbf{t}_{0}, \mathbf{s}_{n+1}\right) \leftarrow p_{1}\left(\mathbf{s}_{1}, \mathbf{t}_{1}\right), \ldots, p_{n}\left(\mathbf{s}_{n}, \mathbf{t}_{n}\right)$ is simplymoded iff $\mathbf{t}_{1}, \ldots, \mathbf{t}_{n}$ is a linear vector of variables and for all $i \in[1, n]$

$$
\operatorname{Var}\left(\mathbf{t}_{i}\right) \cap \operatorname{Var}\left(\mathbf{t}_{0}\right)=\emptyset \text { and } \operatorname{Var}\left(\mathbf{t}_{i}\right) \cap \bigcup_{j=1}^{i} \operatorname{Var}\left(\mathbf{s}_{j}\right)=\emptyset .
$$

A query $\mathbf{B}$ is simply-moded iff the clause $q \leftarrow \mathbf{B}$ is simply-moded, where $q$ is any variable-free atom. A program is simply-moded iff all of its clauses are.

Thus, a clause is simply-moded if the output positions of body atoms are filled in by distinct variables, and every variable occurring in an output position of a body atom does not occur in an earlier input position. In particular, every unit clause is simply-moded. Notice also that programs APPEND and IN_ORDER are simply-moded wrt. the modes append (In, In, Out) and in_order (In, Out).

\section{Input-Consuming Programs}

Input-consuming derivations are a formalism for describing dynamic scheduling in an abstract way [18.

Definition 3.1. A derivation step $\mathbf{A}, B, \mathbf{C} \stackrel{\theta}{\Longrightarrow}(\mathbf{A}, \mathbf{B}, \mathbf{C}) \theta$ is input-consuming iff $\operatorname{In}(B) \theta=\operatorname{In}(B)$. A derivation is input-consuming iff all its derivation steps are input-consuming.

Thus, allowing only input-consuming derivations is a form of dynamic scheduling, since selectability depends on the degree of instantiation at runtime. If no atom is resolvable via an input-consuming derivation step, the query deadlocks 2

It has been shown that the input-consuming resolvent of a simply-moded query using a simply-moded clause is simply-moded [4].

Example 3.2. Consider again the delay declaration

$$
\text { delay append (Ls, _, -) until nonvar(Ls). }
$$

\footnotetext{
${ }^{2}$ Notice that there is a difference between this notion of deadlock and the one used for programs with delay declarations; see [6] for a detailed discussion.
} 
It is easy to check that every derivation starting in a query append $(t, s, \mathrm{X})$, where $\mathrm{X}$ is a variable disjoint from $s$ and $t$, is input-consuming wrt. append ( $\mathrm{In}, \mathrm{In}, \mathrm{ut}$ ) iff it respects the delay declaration.

To show the correspondence between delay declarations and input-consuming derivations suggested by Ex. 3.2, we need some further definitions. We call a term $t$ flat if $t$ has the form $f\left(x_{1}, \ldots, x_{n}\right)$ where the $x_{i}$ are distinct variables. Note that constants are flat terms. The significance of flat term arises from the following observation: if $s$ and $t$ are unifiable, $s$ is non-variable and $t$ is flat, then $s$ is an instance of $t$. Think here of $s$ being a term in an input position of a selected atom, and $t$ being the term in that position of a clause head.

Definition 3.3. A program $P$ is input-consistent iff for each clause $H \leftarrow \mathbf{B}$ of it, the family of terms filling in the input positions of $H$ is linear, and consists of variables and flat terms.

We also consider here delay declarations of a restricted type.

Definition 3.4. A program with delay declarations is simple if every delay declaration is of the form

$$
\text { delay } p\left(\mathrm{X}_{1}, \ldots, \mathrm{X}_{n}\right) \text { until nonvar }\left(\mathrm{X}_{i_{1}}\right) \wedge \ldots \wedge \operatorname{nonvar}\left(\mathrm{X}_{i_{k}}\right)
$$

where $i_{1}, \ldots, i_{k}$ are input positions of $p$.

Moreover, we say that the positions $i_{1}, \ldots, i_{k}$ of $p$ are controlled, while the other input positions of $p$ are free.

Thus the controlled positions are those "guarded" by a delay declaration. The main result of this section shows that, under some circumstances, using delay declarations is equivalent to restricting to input-consuming derivations.

Lemma 3.5. Let $P$ be simply-moded, input-consistent and simple. Let $Q$ be a simply-moded query.

- If for every clause $H \leftarrow \mathbf{B}$ of $P, H$ contains variables in its free positions, then every derivation of $P \cup\{Q\}$ respecting the delay declarations is inputconsuming (modulo renaming).

- If in addition for every clause $H \leftarrow \mathbf{B}$ of $P$, the head $H$ contains flat terms in its controlled positions, then every input-consuming derivation of $P \cup\{Q\}$ respects the delay declarations.

In order to assess how realistic these conditions are, we have checked them against a number of programs from various collections. (The results can be found in Sec. 6). Concerning the statement that all delay-respecting derivations are input-consuming, we are convinced that this is the case in the overwhelming majority of practical cases. Concerning the converse, that is, that all inputconsuming derivations are delay-respecting, we could find different examples in which this was not the case. In many of them this could be fixed by a simple 
transformation of the programs $3^{3}$, in other cases it could not (e.g., flatten, [19]). Nevertheless, we strongly believe that the latter form a small minority.

The delay declarations for the considered programs were either given or derived based on the presumed mode. Note that delay declarations as in Def. 3.4 can be more efficiently implemented than, e.g., delay declarations testing for groundness. Usually, the derivations permitted by the latter delay declarations are a strict subset of the input-consuming derivations.

\section{A Denotational Semantics}

Previous declarative semantics for logic programs cannot correctly model dynamic scheduling. E.g., none of them reflects the fact that append (X, Y, Z) deadlocks. We define a model-theoretic semantics that models computed answer substitutions of input-consuming derivations of simply-moded programs and queries.

We now define simply-local substitutions, which reflect the way clauses become instantiated in input-consuming derivations. A simply-local substitution can be decomposed into several substitutions, corresponding to the instantiation of the output of each body atom, as well as the input of the head.

Definition 4.1. Let $\theta$ be a substitution. We say that $\theta$ is simply-local wrt. the clause $c: p\left(\mathbf{t}_{0}, \mathbf{s}_{n+1}\right) \leftarrow p_{1}\left(\mathbf{s}_{1}, \mathbf{t}_{1}\right), \ldots, p_{n}\left(\mathbf{s}_{n}, \mathbf{t}_{n}\right)$ iff there exist substitutions $\sigma_{0}, \sigma_{1} \ldots, \sigma_{n}$ and disjoint sets of fresh (wrt. $c$ ) variables $v_{0}, v_{1}, \ldots, v_{n}$ such that $\theta=\sigma_{0} \sigma_{1} \cdots \sigma_{n}$ where for $i \in\{0, \ldots, n\}$,

$-\operatorname{Dom}\left(\sigma_{i}\right) \subseteq \operatorname{Var}\left(\mathbf{t}_{i}\right)$,

$-\operatorname{Ran}\left(\sigma_{i}\right) \subseteq \operatorname{Var}\left(\mathbf{s}_{i} \sigma_{0} \sigma_{1} \cdots \sigma_{i-1}\right) \cup v_{i}$

$\theta$ is simply-local wrt. a query $\mathbf{B}$ iff $\theta$ is simply-local wrt. the clause $q \leftarrow \mathbf{B}$ where $q$ is any variable-free atom.

Note that if $\mathbf{A}, B, \mathbf{C} \stackrel{\theta}{\Longrightarrow}(\mathbf{A}, \mathbf{B}, \mathbf{C}) \theta$ is an input-consuming derivation step using clause $c: H \leftarrow \mathbf{B}$, then $\theta_{\mid H}$ is simply-local wrt. the clause $H \leftarrow$ and $\theta_{\mid B}$ is simply-local wrt. the query $B$.

Example 4.2. Consider APPEND in mode append ( $\mathrm{In}, \mathrm{In}, \mathrm{Out}$ ), and its recursive clause $c$ : append $([\mathrm{H} \mid \mathrm{Xs}], \mathrm{Ys},[\mathrm{H} \mid \mathrm{Zs}]) \leftarrow \operatorname{append}(\mathrm{Xs}, \mathrm{Ys}, \mathrm{Zs})$. The substitution $\theta=$ $\{\mathrm{H} / \mathrm{V}, \mathrm{Xs} /[], \mathrm{Ys} /[\mathrm{W}], \mathrm{Zs} /[\mathrm{W}]\}$ is simply-local wrt. $c$ : let $\sigma_{0}=\{\mathrm{H} / \mathrm{V}, \mathrm{Xs} /[], \mathrm{Ys} /[\mathrm{W}]\}$ and $\sigma_{1}=\{\mathrm{Zs} /[\mathrm{W}]\}$; then $\operatorname{Dom}\left(\sigma_{0}\right) \subseteq\{\mathrm{H}, \mathrm{Xs}, \mathrm{Ys}\}$, and $\operatorname{Ran}\left(\sigma_{0}\right) \subseteq v_{0}$ where $v_{0}=$ $\{\mathrm{V}, \mathrm{W}\}$, and $\operatorname{Dom}\left(\sigma_{1}\right) \subseteq\{\mathrm{Zs}\}$, and $\operatorname{Ran}\left(\sigma_{1}\right) \subseteq \operatorname{Var}\left((\mathrm{Xs}, \mathrm{Ys}) \sigma_{0}\right)$.

\footnotetext{
${ }^{3}$ To give an intuitive idea, the transformation would, e.g., replace the clause $\operatorname{even}(\mathrm{s}(\mathrm{s}(\mathrm{X})))$ : - $\operatorname{even}(\mathrm{X})$. with even $(\mathrm{s}(\mathrm{Y}))$ : - $\mathrm{S}_{-} \operatorname{decomp}(\mathrm{Y}, \mathrm{X})$, even $(\mathrm{X})$., where we define $\mathbf{S}_{-} \operatorname{dec} \operatorname{comp}(\mathrm{S}(\mathrm{X}), \mathrm{X})$. and the mode is $\mathbf{S}_{-} \operatorname{decomp}(\mathrm{In}$, Out) .

${ }^{4}$ Note that $\mathbf{s}_{0}$ is undefined. By abuse of notation, $\operatorname{Var}\left(\mathbf{s}_{0} \ldots\right)=\emptyset$.
} 


\subsection{Modelling Complete Derivations}

In predicate logic, an interpretation states which formulas are true and which ones are not. For our purposes, it is convenient to formalise this by defining an interpretation $I$ as a set of atoms closed under variance. Based on this notion and simply-local substitutions, we now define a restricted notion of model.

Definition 4.3. Let $M$ be an interpretation. We say that $M$ is a simply-local model of $c: H \leftarrow B_{1}, \ldots, B_{n}$ iff for every substitution $\theta$ simply-local wrt. $c$,

$$
\text { if } B_{1} \theta, \ldots, B_{n} \theta \in M \text { then } H \theta \in M \text {. }
$$

$M$ is a simply-local model of a program $P$ iff it is a simply-local model of each clause of it.

Note that a simply-local model is not necessarily a model in the classical sense, since the substitution in (1) is required to be simply-local. For example, given the program $\{\mathrm{q}(1), \mathrm{p}(\mathrm{X}) \leftarrow \mathrm{q}(\mathrm{X})\}$ with modes $\mathrm{q}($ In $), \mathrm{p}($ Out $)$, a model must contain the atom $\mathrm{p}(1)$, whereas a simply-local model does not necessarily contain $\mathrm{p}(1)$, since $\{\mathrm{X} / 1\}$ is not simply-local wrt. $\mathrm{p}(\mathrm{X}) \leftarrow \mathrm{q}(\mathrm{X})$.

We now show that there exists a minimal simply-local model and that it is bottom-up computable. For this we need the following operator $T_{P}^{S L}$ on interpretations: Given a program $P$ and an interpretation $I$, define

$$
\left.\begin{array}{rl}
T_{P}^{S L}(I)=\{H \theta \mid & \exists c: H \leftarrow B_{1}, \ldots, B_{n} \in P \\
& \exists \theta \text { simply-local wrt.c } \\
& B_{1}, \ldots, B_{n} \theta \in I
\end{array}\right\} .
$$

Operator's powers are defined in the standard way: $T_{P}^{S L} \uparrow 0(I)=I, T_{P}^{S L} \uparrow$ $(i+1)(I)=T_{P}^{S L}\left(T_{P}^{S L} \uparrow i(I)\right)$, and $T_{P}^{S L} \uparrow \omega(I)=\bigcup_{i=0}^{\infty} T_{P}^{S L} \uparrow i(I)$. It is easy to show that $T_{P}^{S L}$ is continuous on the lattice where interpretations are ordered by set inclusion. Hence, by well-known results, $T_{P}^{S L} \uparrow \omega$ exists and is the least fixpoint of $T_{P}^{S L}$. We can now state our main result.

Theorem 4.4. Let $P$ be simply-moded. Then $T_{P}^{S L} \uparrow \omega(\emptyset)$ is the least simplylocal model of $P$.

We now prove correctness, fully abstractness and compositionality of the semantics. We denote the least simply-local model of $P$ by $M_{P}^{S L}$.

Theorem 4.5. Let the program $P$ and the query $\mathbf{A}$ be simply-moded. The following statements are equivalent:

(i) there exists an input-consuming successful derivation $\mathbf{A} \stackrel{\vartheta}{\longrightarrow} P \square$,

(ii) there exists a substitution $\theta$, simply-local wrt. $\mathbf{A}$, such that $\mathbf{A} \theta \in M_{P}^{S L}$,

where $\mathbf{A} \theta$ is a variant of $\mathbf{A} \vartheta$. 
Example 4.6. Considering again APPEND, we have that $M_{\mathrm{APPEND}}^{S L}=\bigcup_{n=0}^{\infty}\left\{\operatorname{append}\left(\left[t_{1}, \ldots, t_{n}\right], s,\left[t_{1}, \ldots, t_{n} \mid s\right]\right) \mid t_{1}, \ldots, t_{n}, s\right.$ are any terms $\}$.

Using Thm. 4.5, we can conclude that the query append ( $[\mathrm{a}, \mathrm{b}], \mathrm{X}, \mathrm{Y}$ ) succeeds with computed answer $\theta=\{\mathrm{Y} /[\mathrm{a}, \mathrm{b} \mid \mathrm{X}]\}$. In fact, $\operatorname{append}([\mathrm{a}, \mathrm{b}], \mathrm{X},[\mathrm{a}, \mathrm{b} \mid \mathrm{X}]) \in$ $M_{\mathrm{APPEND}}^{S L}$, and $\theta$ is simply-local wrt. the query above.

On the other hand, we can also say that the query append (X, $[\mathrm{a}, \mathrm{b}], \mathrm{Y})$ has no successful input-consuming derivations. In fact, for every $A \in M_{\mathrm{APPEND}}^{S L}$ we have that the first input position of $A$ is filled in by a non-variable term. Therefore there is no simply-local $\theta$ such that append $(\mathrm{X},[\mathrm{a}, \mathrm{b}], \mathrm{Y}) \theta \in M_{\mathrm{APPEND}}^{S L}$. This shows that this semantics allows us to model correctly deadlocking derivations.

However, append (X, $[\mathrm{a}, \mathrm{b}], \mathrm{Y}$ ) has instances in $M_{\mathrm{APPEND}}^{S L}$, and successful derivations, if the requirement of simply-local substitutions, resp. input-consuming derivations, is ignored.

\subsection{Modelling Partial Derivations}

Dynamic scheduling also allows for parallelism. In this context it is important to be able to model the result of partial derivations. That is to say, instead of considering computed answer substitutions for complete derivations, we now consider computed answer substitutions for partial derivations. As we will see, this will be essential in order to prove termination of the programs.

Let $S M_{P}$ be the set of all simply-moded atoms of the extended Herbrand universe of $P$. In analogy to Theorem 4.4, we have the following theorem.

Theorem 4.7. Let $P$ be simply-moded. Then $T_{P}^{S L} \uparrow \omega\left(S M_{P}\right)$ is the least simply-local model of $P$ containing $S M_{P}$.

We denote the least simply-local model of $P$ containing $S M_{P}$ by $P M_{P}^{S L}$, for partial model. We now show correctness, fully abstractness and compositionality of this semantics for partial derivations.

Theorem 4.8. Let the program $P$ and the query $\mathbf{A}$ be simply-moded. The following statements are equivalent:

(i) there exists an input-consuming derivation $\mathbf{A} \stackrel{\vartheta}{\longrightarrow} P \mathbf{A}^{\prime}$,

(ii) there exists a substitution $\theta$, simply-local wrt. $\mathbf{A}$, such that $\mathbf{A} \theta \in P M_{P}^{S L}$,

where $\mathbf{A} \theta$ is a variant of $\mathbf{A} \vartheta$.

Note that the derivation in point (i) ends in $\mathbf{A}^{\prime}$, which might be non-empty.

Example 4.9. Consider again APPEND. First, $P M_{\mathrm{APPEND}}^{S L}$ contains $M_{\mathrm{APPEND}}^{S L}$ as a subset (see Ex. 4.6). Note that $M_{\mathrm{APPEND}}^{S L}$ is obtained by starting from the fact clause append ([], Ys, Ys) and repeatedly applying the $T_{P}^{S L}$ operator using the recursive clause of APPEND. Now to obtain the remaining atoms in $P M_{\mathrm{APPEND}}^{S L}$, we must 
repeatedly apply the $T_{P}^{S L}$ operator, starting from any simply moded atom, i.e., an atom of the form append $(s, t, x)$ where $s$ and $t$ are arbitrary terms but $x$ does not occur in $s$ or $t$. It is easy to see that we thus have to add $S M_{P}$ together with

$$
\begin{aligned}
& \left\{\operatorname{append}\left(\left[t_{1}, \ldots, t_{n} \mid s\right], t,\left[t_{1}, \ldots, t_{n} \mid x\right]\right) \mid t_{1}, \ldots, t_{n}, s, t\right. \text { are arbitrary terms, } \\
& x \text { is a fresh variable }\} \text {. }
\end{aligned}
$$

Using Thm. 4.8 we can conclude that the query append ( $[\mathrm{a}, \mathrm{b} \mid \mathrm{X}], \mathrm{Y}, \mathrm{Z})$ has a partial derivation with computed answer $\theta=\left\{\mathrm{Z} /\left[\mathrm{a}, \mathrm{b} \mid \mathrm{Z}^{\prime}\right]\right\}$, and indeed, append $\left([\mathrm{a}, \mathrm{b} \mid \mathrm{X}], \mathrm{Y},\left[\mathrm{a}, \mathrm{b} \mid \mathrm{Z}^{\prime}\right]\right) \in P M_{\mathrm{APPEND}}^{S L}$, and $\theta$ is simply-local wrt. the query above. Notice that, following the same reasoning, one can also conclude that the query also has a partial derivation with computed answer $\theta=\left\{\mathrm{Z} /\left[\mathrm{a} \mid \mathrm{Z}^{\prime}\right]\right\}$.

\section{Termination}

Input-consuming derivations were originally conceived as an abstract and "reasonably strong" assumption about the selection rule in order to prove termination [18. The first result in this area was a sufficient criterion applicable to well- and nicely-moded programs. This was improved upon by dropping the requirement of well-modedness, which means that one also captures termination by deadlock 6. In this section, we only consider simply moded programs and queries (simply-moded and well-moded programs form two largely overlapping, but distinct classes), and we provide a criterion for termination which is sufficient and necessary, and hence an exact characterisation of termination. We first define our notion of termination.

Definition 5.1. A program is input terminating iff all its input-consuming derivations started in a simply-moded query are finite.

In order to prove that a program is input terminating we need the concept of moded level mapping [10].

Definition 5.2. A function || is a moded level mapping iff it maps atoms into $\mathbb{N}$ and such that for any $\mathbf{s}, \mathbf{t}$ and $\mathbf{u},|p(\mathbf{s}, \mathbf{t})|=|p(\mathbf{s}, \mathbf{u})|$.

The condition $|p(\mathbf{s}, \mathbf{t})|=|p(\mathbf{s}, \mathbf{u})|$ states that the level of an atom is independent from the terms in its output positions.

Note that programs without recursion terminate trivially. In this context, we need the following standard definitions [2].

Definition 5.3. Let $P$ be a program, $p$ and $q$ be relations. We say that

- $p$ refers to $q$ iff there is a clause in $P$ with $p$ in the head and $q$ in the body.

- $p$ depends on $q$ iff $(p, q)$ is in the reflexive and transitive closure of the relation refers to.

- $p$ and $q$ are mutually recursive, written $p \simeq q$, iff $p$ and $q$ depend on each other. 
We now define simply-acceptability, which is in analogy to acceptability [5], but defined to deal with simply-moded and input-consuming programs.

Definition 5.4. Let $P$ be a program and $M$ a simply-local model of $P$ containing $S M_{P}$. A clause $H \leftarrow \mathbf{A}, B, \mathbf{C}$ is simply-acceptable wrt. the moded level mapping || and $M$ iff for every substitution $\theta$ simply-local wrt. it,

$$
\text { if } \mathbf{A} \theta \in M \text { and } \operatorname{Rel}(H) \simeq \operatorname{Rel}(B) \text { then }|H \theta|>|B \theta| \text {. }
$$

The program $P$ is simply-acceptable wrt. $M$ iff there exists a moded level mapping || such that each clause of $P$ is simply-acceptable wrt. || and $M$.

We also say that $P$ is simply-acceptable if it is simply acceptable wrt. some $M$. We can now show that this concept allows to characterize the class of input terminating programs.

Theorem 5.5. A simply-moded program $P$ is simply-acceptable iff it is input terminating. In particular, if $P$ is input terminating, then it is simply-acceptable wrt. $P M_{P}^{S L}$.

Let us compare simply-acceptability to acceptability, used to prove lefttermination 5]. Acceptability is based on a (classical) model $M$ of the program, and for a clause $H \leftarrow A_{1}, \ldots, A_{n}$, one requires $|H \theta|>\left|A_{i} \theta\right|$ only if $M \models\left(A_{1}, \ldots, A_{i-1}\right) \theta$. The reason is that for LD-derivations, $A_{1}, \ldots, A_{i-1}$ must be completely resolved before $A_{i}$ is selected. By the correctness of LD resolution [2], it turns out that the c.a.s. $\theta$, just before $A_{i}$ is selected, is such that $M \models\left(A_{1}, \ldots, A_{i-1}\right) \theta$. It has been argued previously that it is difficult to use a similar argument for input-consuming derivations [18]. Using the results of the previous section, we have overcome this problem. We exploited that provided that programs and queries are simply-moded, we know that even though $A_{1}, \ldots, A_{i-1}$ may not be resolved completely, $A_{1}, \ldots, A_{i-1} \theta$ will be in any "partial model" of the program.

Example 5.6. Figure1] shows program 15.3 from [19]: quicksort using a form of difference lists (we permuted two body atoms for the sake of clarity). This program is simply-moded, and when used in combination with dynamic scheduling, the standard delay declarations for it are the following:

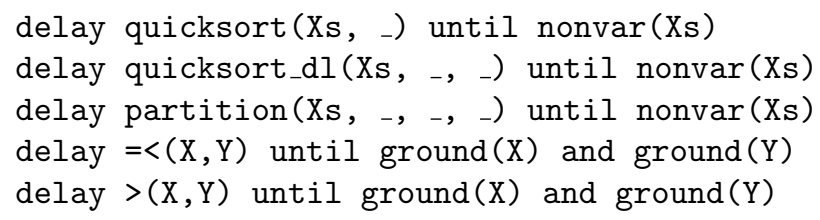

The last two declarations fall out of the scope of Lemma 3.5. Nevertheless, if we think of the built-ins $>$ and $=<$ as being conceptually defined by a program containing infinitely many ground facts of the form $>(n, m)$, with $n$ and $m$ being two appropriate integers, the derivations respecting the above delay declarations 
$\%$ quicksort $(\mathrm{Xs}, \mathrm{Ys}) \leftarrow \mathrm{Ys}$ is an ordered permutation of $\mathrm{Xs}$.

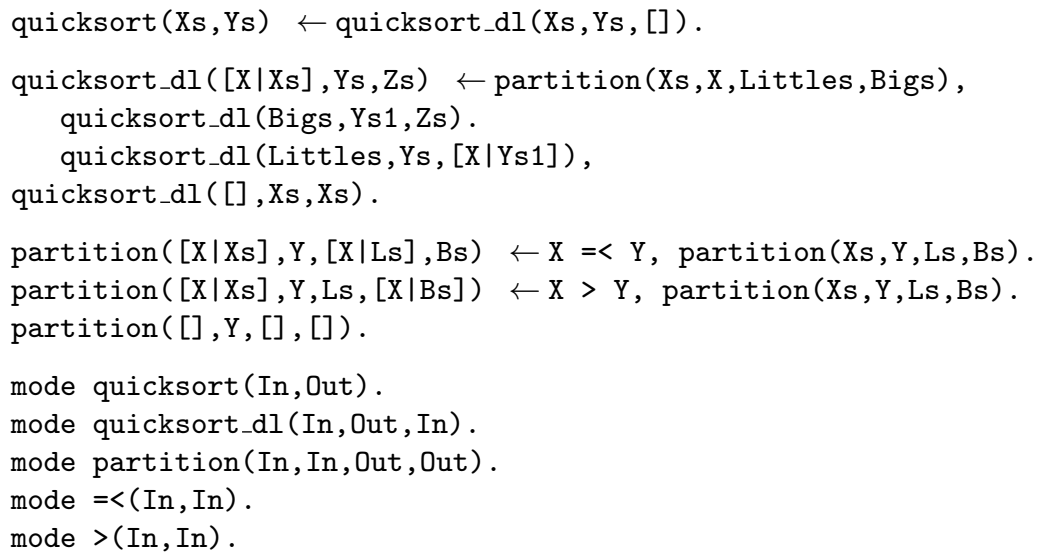

Fig. 1. The quicksort program

are exactly the input-consuming ones. We can prove that the program is input terminating. Define len as

$$
\begin{aligned}
\operatorname{len}([h \mid t]) & =1+\operatorname{len}(t), \\
\operatorname{len}(a) & =0
\end{aligned} \quad \text { if } a \text { is not of the form }[h \mid t] .
$$

We use the following moded level mapping (positions with _ are irrelevant)

$$
\begin{aligned}
& \mid \text { quicksort_dl }\left(l,,_{-},-\right) \mid=\operatorname{len}(l) \text {, } \\
& \left|\operatorname{partition}\left(l,,_{-},{ }_{-},-\right)\right|=\operatorname{len}(l) \text {. }
\end{aligned}
$$

The level mapping of all other atoms can be set to 0 . Concerning the model, the simplest solution is to use the model that expresses the dependency between the list lengths of the arguments of partition, i.e., $M$ should contain all atoms of the form partition $\left(l_{1}, x, l_{2}, l_{3}\right)$ where $\operatorname{len}\left(l_{1}\right)>\operatorname{len}\left(l_{2}\right)$ and $\operatorname{len}\left(l_{1}\right)>\operatorname{len}\left(l_{3}\right)$.

\section{Benchmarks}

In order to assess how realistic the conditions of Lemma 3.5 are, we have looked into three collections of logic programs, and we have checked whether those programs were simply moded (SM), input-consistent (IC) and whether they satisfied both sides of Lemma 3.5 (L). Notice that programs which are not inputconsistent do not satisfy the conditions of Lemma 3.5. For this reason, some $\mathbf{L}$ columns are left blank. The results, reported in Tables 1 to 3 show that our results apply to the majority of the programs considered. We considered in Table 1 the programs from Apt's collection [25], in Table 2 those of the DPPD's collection, (http://dsse.ecs.soton.ac.uk/ mal/systems/dppd.html), and in Table 3 some programs of Lindenstrauss's collection (http://www.cs.huji.ac.il/ naomil). 
Table 1. Programs from Apt's Collection

\begin{tabular}{|c|c|c|c|c|c|c|c|}
\hline & SM & IC & $\mathbf{L}$ & & SM & IC & $\mathbf{L}$ \\
\hline append (In, In, Out) & yes & yes & yes & mergesort(In, Out) & yes & no & \\
\hline append (Out, Out, In) & yes & yes & no & mergesort (Out, In) & no & & \\
\hline append3 (In, In, In, Out) & yes & yes & yes & mergesort_variant (In, Out, In) & yes & yes & no \\
\hline color_map (In, Out) & yes & no & & ordered(In) & yes & no & \\
\hline color_map (Out, In) & yes & yes & yes & overlap (In, In) & yes & no & \\
\hline dcsolve (In,_) & yes & yes & yes & overlap (In, Out) & yes & yes & yes \\
\hline even (In) & yes & no & & overlap (Out, In) & yes & yes & yes \\
\hline fold (In, In, Out) & yes & yes & yes & perm_select (In, Out) & yes & yes & no \\
\hline list (In) & yes & yes & yes & perm_select(Out, In) & yes & yes & no \\
\hline lte (In, In) & yes & yes & no & qsort (In, Out) & yes & yes & yes \\
\hline Ite (In, Out) & yes & yes & yes & qsort(Out, In) & no & & \\
\hline Ite(Out,In) & yes & yes & no & reverse(In, Out) & yes & yes & yes \\
\hline $\operatorname{map}(\operatorname{In}, \operatorname{In})$ & yes & yes & yes & reverse(Out, In) & yes & yes & yes \\
\hline $\operatorname{map}(\mathrm{In}$, Out) & yes & yes & yes & select (In, In, Out) & yes & no & \\
\hline $\operatorname{map}$ (Out, In) & yes & yes & yes & select (Out, In, Out) & yes & yes & yes \\
\hline member (In, In) & yes & no & & subset $(\operatorname{In}, \operatorname{In})$ & yes & no & \\
\hline member (In, Out) & yes & yes & yes & subset (Out, In) & yes & yes & yes \\
\hline member (Out, In) & yes & yes & yes & $\operatorname{sum}(\operatorname{In}$, In , Out) & yes & yes & yes \\
\hline type (In, In, Out) & no & & & sum (Out, Out, In) & yes & yes & yes \\
\hline
\end{tabular}

\section{Conclusion}

In this paper, we have proven a result that demonstrates - for a large class of programs - the equivalence between delay declarations and input-consuming derivations. This was only speculated in [6,7]. In fact, even though the class of programs we are considering here (simply-moded programs) is only slightly smaller than the one of nicely-moded programs considered in [67], for the latter a result such as Lemma 3.5 does not hold.

We have provided a denotational semantics for input-consuming derivations using a variant of the well-known $T_{P}$-operator. Our semantics follows the $s$-semantics approach [9] and thus enjoys the typical properties of semantics in this class. This semantics improves on the one introduced in [7] in two respects: The semantics of this paper models (within a uniform framework) both complete and incomplete derivations, and there is no requirement that the program must be well-moded.

Falaschi et al. 11] have defined a denotational semantics for CLP programs with dynamic scheduling of a somewhat different kind: the semantics of a query is given by a set of closure operators; each operator is a function modelling a possible effect of resolving the query on a program state (i.e., constraint on the program variables). However, we believe that our approach is more suited to termination proofs. 
Table 2. Programs from DPPD's Collection

\begin{tabular}{|c|c|c|c|c|c|c|c|}
\hline & SM & IC & $\mathbf{L}$ & & $\mathbf{S M}$ & IC & $\mathbf{L}$ \\
\hline applast (In, In, & yes & yes & yes & relative (In,Out) & yes & yes & yes \\
\hline $\operatorname{depth}($ In , Out) & yes & no & & relative (Out, In) & yes & yes & ? \\
\hline flipflip(In,Out) & yes & yes & yes & rev_acc (In, In, Out) & yes & yes & 1 \\
\hline flipflip(Out,In) & yes & yes & yes & rotate(In,Out) & yes & yes & \\
\hline generate (In, In, Out) & yes & no & & rotate(Out, In) & yes & yes & $\mathrm{ye}$ \\
\hline liftsolve(In, In) & yes & yes & yes & solve(In, In, Out) ) & yes & no & \\
\hline liftsolve(In,Out) & yes & yes & yes & square_square (In, Out) & yes & yes & es \\
\hline match (In, In) & yes & no & & squretr (In, Out) & yes & yes & yes \\
\hline match_app (In, In) & yes & yes & no & ssupply (In, In, Out) & yes & yes & e \\
\hline match_app (In, Out) & yes & yes & no & trace (In, In, Out) & yes & no & \\
\hline max_lenth(In,Out,Out) & yes & yes & yes & trace (In, Out, Out) & no & & \\
\hline memo_solve(In, Out) & yes & no & & transpose (In, Out) & yes & no & \\
\hline prune (In, Out) & yes & no & & transpose (Out, In) & yes & yes & ye \\
\hline orune (Out, In) & yes & no & & unify (In, In, Out) & yes & no & \\
\hline
\end{tabular}

Table 3. Programs from Lindenstrauss's Collection

\begin{tabular}{|c|c|c|c|c|c|c|c|}
\hline & $\mathbf{S M}$ & IC & $\mathbf{L}$ & & SM & IC & $\mathbf{L}$ \\
\hline $\operatorname{ack}\left(\operatorname{In}, \operatorname{In},{ }_{-}\right)$ & yes & yes & no & huffman (In, Out) & no & & \\
\hline concatenate (In, In, Out) & yes & yes & yes & huffman (In, Out) & no & & \\
\hline credit(In, Out) & yes & yes & yes & normal_form (_, In) & yes & no & \\
\hline $\operatorname{deep}($ In, Out $)$ & yes & yes & yes & queens (In, Out) & yes & yes & yes \\
\hline deep (Out, In) & no & & & queens (Out, In) & yes & yes & no \\
\hline descendant (In, Out) & yes & yes & yes & rewrite(In, Out) & yes & no & \\
\hline descendant (Out, In) & yes & yes & yes & transf orm (In, In, In, Out) & yes & yes & yes \\
\hline holds (In, Out) & yes & yes & yes & twoleast (In, Out) & no & & \\
\hline
\end{tabular}

As mentioned in Sec. 4.2 in the context of parallelism and concurrency [17, one can have derivations that never succeed, and yet compute substitutions. Moreover, input-consuming derivations essentially correspond to the execution mechanism of (Moded) FGHC [20]. Thus we have provided a model-theoretic semantics for such programs/programming languages, which go beyond the usual success-based SLD resolution mechanism of logic programming.

On a more practical level, our semantics for partial derivations is used in order to prove termination. We have provided a necessary and sufficient criterion for termination, applicable to a wide class of programs, namely the class of simplymoded programs. For instance, we can now prove the termination of QUICKSORT, which is not possible with the tools of [18,6] (which provided only a sufficient condition). In the termination proofs, we exploit that any selected atom in an input-consuming derivation is in a model for partial derivations, in a similar way as this is done for proving left-termination. It is only on the basis of the semantics that we could present a characterisation of input-consuming termination for simply-moded programs. 


\section{References}

1. K. R. Apt. Introduction to Logic Programming. In J. van Leeuwen, editor, Handbook of Theoretical Computer Science, volume B: Formal Models and Semantics, pages 495-574. Elsevier, Amsterdam and The MIT Press, Cambridge, 1990.

2. K. R. Apt. From Logic Programming to Prolog. Prentice Hall, 1997.

3. K. R. Apt and S. Etalle. On the unification free Prolog programs. In A. Borzyszkowski and S. Sokolowski, editors, Proceedings of MFCS '93, LNCS, pages 1-19. Springer-Verlag, 1993.

4. K. R. Apt and I. Luitjes. Verification of logic programs with delay declarations. In V. S. Alagar and M. Nivat, editors, Proceedings of AMAST'95, LNCS, pages 66-90. Springer-Verlag, 1995. Invited Lecture.

5. K. R. Apt and D. Pedreschi. Modular termination proofs for logic and pure Prolog programs. In G. Levi, editor, Advances in Logic Programming Theory, pages 183229. Oxford University Press, 1994.

6. A. Bossi, S. Etalle, and S. Rossi. Properties of input-consuming derivations. ENTCS, 30(1), 1999. http://www.elsevier.nl/locate/entcs.

7. A. Bossi, S. Etalle, and S. Rossi. Semantics of input-consuming programs. In J. Lloyd, editor, CL 2000. Springer-Verlag, 2000.

8. A. Bossi, S. Etalle, S. Rossi, and J.-G. Smaus. Semantics and termination of simply-moded logic programs with dynamic scheduling. Available via CoRR: http://arXiv.org/archive/cs/intro.html, 2001.

9. A. Bossi, M. Gabbrielli, G. Levi, and M. Martelli. The $s$-semantics approach: theory and applications. Journal of Logic Programming, 19/20:149-197, 1994.

10. S. Etalle, A. Bossi, and N. Cocco. Termination of well-moded programs. Journal of Logic Programming, 38(2):243-257, 1999.

11. M. Falaschi, M. Gabbrielli, K. Marriott, and C. Palamidessi. Constraint logic programming with dynamic scheduling: A semantics based on closure operators. Information and Computation, 137:41-67, 1997.

12. P. M. Hill and J. W. Lloyd. The Gödel Programming Language. The MIT Press, 1994.

13. Intelligent Systems Laboratory, Swedish Institute of Computer Science, PO Box 1263, S-164 29 Kista, Sweden. SICStus Prolog User's Manual, 1998. http://www.sics.se/sicstus/docs/3.7.1/html/sicstus_toc.html.

14. R. A. Kowalski. Algorithm $=$ Logic + Control. Communications of the ACM, 22(7):424-436, 1979.

15. J. W. Lloyd. Foundations of Logic Programming. Symbolic Computation - Artificial Intelligence. Springer-Verlag, 1987.

16. L. Naish. Negation and Control in Prolog, volume 238 of LNCS. Springer-Verlag, 1986.

17. L. Naish. Parallelizing NU-Prolog. In R. A. Kowalski and K. A. Bowen, editors, Proceedings of ICLP/SLP '88, pages 1546-1564. MIT Press, 1988.

18. J.-G. Smaus. Proving termination of input-consuming logic programs. In D. De Schreye, editor, Proceedings of ICLP'99, pages 335-349. MIT Press, 1999.

19. L. Sterling and E. Shapiro. The Art of Prolog. MIT Press, 1986.

20. K. Ueda and M. Morita. Moded Flat GHC and its message-oriented implementation technique. New Generation Computing, 13(1):3-43, 1994. 